\title{
Asymptotic Lattices and W-Congruences in Integrable Discrete Geometry
}

\author{
Adam DOLIWA \\ Instytut Fizyki Teoretycznej, Uniwersytet Warszawski, \\ ul. Hoża 69, 00-681 Warszawa, Poland \\ E-mail: doliwa@fuw.edu.pl
}

\begin{abstract}
The asymptotic lattices and their transformations are included into the theory of quadrilateral lattices.
\end{abstract}

\section{Introduction}

The multidimensional lattices of planar quadrilaterals (quadrilateral lattices) seem to be the basic concept of the geometric theory of discrete integrable systems. The detailed investigation of such lattices, their transformations and reductions was the subject of intensive studies during last few years $[1,6,11,4,7,17,12,9,16,8,10]$. However, the theory of asymptotic lattices $[2,3]$ had to be treated as separate issue in the integrable discrete geometry. In a recent work [5] it was shown that the asymptotic lattices, and their Darboux-type transformations given by the Weingarten (or $\mathrm{W}$ - for short) congruences, form a part of the theory of the quadratic reductions of the quadrilateral lattices [8]. It should be noted here that both two-dimensional discrete conjugate nets and asymptotic lattices have been defined long time ago [20] without any relation to integrable systems, as convenient approximations of the corresponding differential-geometric coordinate systems on surfaces.

The paper is constructed as follows. In Section 2 we collect basic results of the Plücker line geometry, which provides the appropriate setting for subsequent discussion of geometric properties of the assymptotic nets and $\mathrm{W}$-congruences. In Section 3 we recall necessary material from the theory of quadrilateral lattices, their transformations and reductions. Finally, in Section 4 we present the theory of asymptotic lattices and $\mathrm{W}$-congruences.

\section{The Plücker line geometry}

Given two different points $[\boldsymbol{u}],[\boldsymbol{v}]$ of $\mathbb{P}^{3}$, the line $\langle[\boldsymbol{u}],[\boldsymbol{v}]\rangle$ passing through them can be represented, up to proportionality factor, by a bi-vector [14]

$$
\mathfrak{p}=\boldsymbol{u} \wedge \boldsymbol{v} \in \bigwedge^{2}\left(\mathbb{R}^{4}\right)
$$


The space of straight lines in $\mathbb{P}^{3}$ can be therefore identified with a subset of $\mathbb{P}\left(\bigwedge^{2}\left(\mathbb{R}^{4}\right)\right) \simeq$ $\mathbb{P}^{5}$; the necessary and sufficient condition for a non-zero bi-vector $\mathfrak{p}$ in order to represent a straight line is given by the homogeneous equation

$$
\mathfrak{p} \wedge \mathfrak{p}=0 .
$$

Given the basis $\boldsymbol{e}_{1}, \ldots, \boldsymbol{e}_{4}$ of $\mathbb{R}^{4}$, then the bi-vectors $\boldsymbol{e}_{i_{1} i_{2}}=\boldsymbol{e}_{i_{1}} \wedge \boldsymbol{e}_{i_{2}}, 1 \leq i_{1}<i_{2} \leq 4$, form the corresponding basis of $\bigwedge^{2}\left(\mathbb{R}^{4}\right)$ :

$$
\mathfrak{p}=p^{12} \boldsymbol{e}_{12}+p^{13} \boldsymbol{e}_{13}+\cdots+p^{34} \boldsymbol{e}_{34} .
$$

Equation (2) rewritten in the Plücker (or Grassmann-Plücker) coordinates $p^{i j}$ reads

$$
p^{12} p^{34}-p^{13} p^{24}+p^{14} p^{23}=0,
$$

and defines in $\mathbb{P}^{5}$ the so-called Plücker (or Plücker-Klein) quadric $\mathcal{Q}_{P}$.

If two lines intersect then the bi-vectors $\mathfrak{p}_{i}, i=1,2$, corresponding to them satisfy not only equations of the form (2), but also

$$
\mathfrak{p}_{1} \wedge \mathfrak{p}_{2}=0,
$$

i.e., the line joining points $\left[\mathfrak{p}_{1}\right],\left[\mathfrak{p}_{2}\right]$ is contained in the Plücker quadric $\mathcal{Q}_{P}$. Therefore, isotropic lines of $\mathbb{P}^{5}$ correspond to planar pencils of lines in $\mathbb{P}^{3}$.

\section{Quadrilateral lattices and congruences}

Definition 1 ([20]). By two dimensional quadrilateral lattice we mean mapping of $\mathbb{Z}^{2}$ in $\mathbb{P}^{M}$, such that its elementary quadrilaterals are planar.

This geometric characterization implies linear relation between homogeneous coordinates $\boldsymbol{y} \in \mathbb{R}^{M+1}$ of four points of any elementary quadrilateral. Such a relation can be put into the form of the discrete Laplace equation

$$
\Delta_{1} \Delta_{2} \boldsymbol{y}=a \Delta_{1} \boldsymbol{y}+b \Delta_{2} \boldsymbol{y}+c \boldsymbol{y}
$$

where $T_{i}, i=1,2$, stands for the shift operator along $i$-th direction of the lattice, and $\Delta_{i}=T_{i}-1$, is the corresponding partial difference operator.

Intersections of tangent lines of the lattice define two new quadrilateral lattices called their Laplace transforms.

Remark. Restriction from $\mathbb{P}^{M}$ to its affine part, and therefore from homogeneous coordinates to non-homogeneous ones, results in putting $c=0$ in equation (5).

The tangents of the lattice are canonical examples of special two-parameter families of straight lines called discrete congruences.

Definition 2 ([12]). $\mathbb{Z}^{2}$-parameter family of lines in $\mathbb{P}^{M}$ is called two dimensional discrete congruence if any two neighbouring lines are coplanar. Intersection points of lines of a discrete congruence with its nearest neighbours in the $i$-th direction form the $i$-th focal lattice of the congruence.

One can show that focal lattices of two dimensional congruences are quadrilateral lattices.

Given a quadric hypersurface of the projective space, then one can show [8] that the quadrilateral lattices with points in such a quadric provide an integrable reduction (the so called quadratic reduction) of the quadrilateral lattice. 


\section{Asymptotic lattices and $\mathrm{W}$-congruences}

Definition 3 ([20]). An asymptotic lattice is a mapping $\boldsymbol{x}: \mathbb{Z}^{2} \mapsto \mathbb{P}^{3}$ such that any point $\boldsymbol{x}$ of the lattice is coplanar with its four nearest neighbours $T_{1} \boldsymbol{x}, T_{2} \boldsymbol{x}, T_{1}^{-1} \boldsymbol{x}$ and $T_{2}^{-1} \boldsymbol{x}$.

Remark. Although the above definition is projectively invariant, we will use the affine notation, i.e., $\boldsymbol{x} \in \mathbb{R}^{3}$ and $[(\boldsymbol{x}, 1)]$ are the corresponding homogeneous coordinates in $\mathbb{P}^{3}$.

The plane in Definition 3 can be called the tangent plane of the asymptotic lattice in the point $\boldsymbol{x}$.

One can express the asymptotic lattice condition in the form of the linear equations

$$
\begin{aligned}
& \Delta_{1} \tilde{\Delta}_{1} \boldsymbol{x}=a_{1} \Delta_{1} \boldsymbol{x}+b_{1} \Delta_{2} \boldsymbol{x}, \\
& \Delta_{2} \tilde{\Delta}_{2} \boldsymbol{x}=a_{2} \Delta_{1} \boldsymbol{x}+b_{2} \Delta_{2} \boldsymbol{x},
\end{aligned}
$$

where $\tilde{\Delta}_{i}=1-T_{i}^{-1}, i=1,2$, is the backward partial difference operator.

The asymptotic tangent lines can be represented in the line geometry by the following bi-vectors

$$
\mathfrak{p}_{i}=\left(\begin{array}{c}
\boldsymbol{x} \\
1
\end{array}\right) \wedge\left(\begin{array}{c}
\Delta_{i} \boldsymbol{x} \\
0
\end{array}\right), \quad i=1,2 .
$$

Notice that the lines $\left\langle\mathfrak{p}_{1}, \mathfrak{p}_{2}\right\rangle$ are generators of the Plücker quadric (both asymptotic tangents intersect in $\boldsymbol{x}$ ) and represent pairs $(\boldsymbol{x}, \pi)$, where $\pi$ is the tangent plane of the asymptotic lattice at the point $\boldsymbol{x}$. Two neighbouring tangent planes $\pi$ and $T_{i} \pi, i=1,2$, intersect along the tangent line represented by $\mathfrak{p}_{i}$ (see Fig. 1). We have thus the following result [5]:

Theorem 1. A discrete asymptotic net in $\mathbb{P}^{3}$ viewed as the envelope of its tangent planes corresponds to a congruence of generators of the Plücker quadric $\mathcal{Q}_{P}$; the focal lattices of the congruence represent asymptotic directions of the lattice.

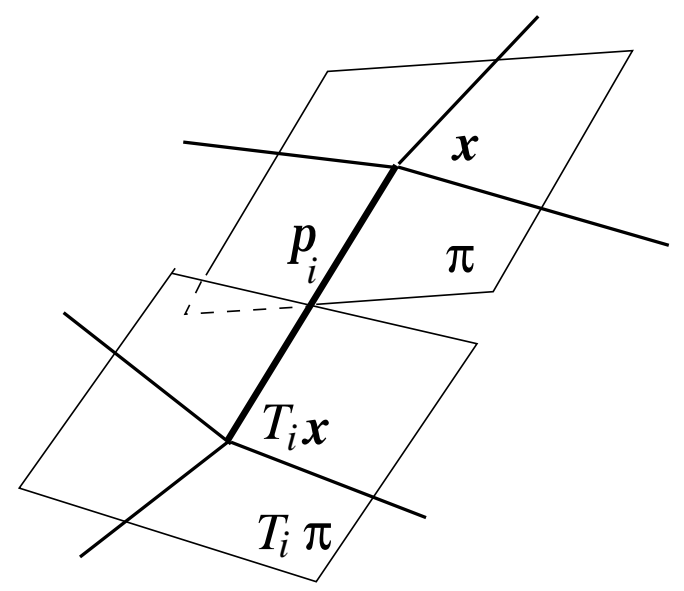

Figure 1. Asymptotic tangents

Corollary 2. The lattices in $\mathcal{Q}_{P}$ which represent two families of asymptotic tangents of an asymptotic lattice are Laplace transforms of each other. 
One can show (for details see $[15,18]$ ) that there exists a discrete analogue of the Lelieuvre representation of asymptotic nets [13]

$$
\begin{aligned}
& \Delta_{1} \boldsymbol{x}=\Delta_{1} \boldsymbol{N} \times \boldsymbol{N}, \\
& \Delta_{2} \boldsymbol{x}=\boldsymbol{N} \times \Delta_{2} \boldsymbol{N},
\end{aligned}
$$

where the vector $\boldsymbol{N}$, orthogonal to the tangent plane of the lattice, satisfies the discrete Moutard equation (see also [19])

$$
T_{1} T_{2} \boldsymbol{N}+\boldsymbol{N}=Q\left(T_{1} \boldsymbol{N}+T_{2} \boldsymbol{N}\right) .
$$

Given a solution $\Theta$ of the discrete Moutard equation (10), one can define the (discrete analogue of the) Moutard transformation [18] (see also [19]) via the linear system

$$
\begin{aligned}
& \Delta_{1}(\Theta \widehat{\boldsymbol{N}})=\left(\Delta_{1} \Theta\right) \boldsymbol{N}-\Theta \Delta_{1} \boldsymbol{N}, \\
& \Delta_{2}(\Theta \widehat{\boldsymbol{N}})=-\left(\Delta_{2} \Theta\right) \boldsymbol{N}+\Theta \Delta_{2} \boldsymbol{N},
\end{aligned}
$$

which implies that $\widehat{\boldsymbol{N}}$ satisfies the Moutard equation (10) with the new proportionality factor

$$
\widehat{Q}=\frac{T_{1} T_{2} \widehat{\Theta}+\widehat{\Theta}}{T_{1} \widehat{\Theta}+T_{2} \widehat{\Theta}}, \quad \widehat{\Theta}=\frac{1}{\Theta} .
$$

The lattice [5]

$$
\widehat{\boldsymbol{x}}=\boldsymbol{x}+\widehat{\boldsymbol{N}} \times \boldsymbol{N}
$$

is a new asymptotic lattice with normal vector $\widehat{\boldsymbol{N}}$. The line $\langle\boldsymbol{x}, \widehat{\boldsymbol{x}}\rangle$ is tangent to both lattices, therefore we have

$$
\Theta \widehat{\boldsymbol{N}} \times \boldsymbol{N}=A \Delta_{1} \boldsymbol{x}+B \Delta_{2} \boldsymbol{x}
$$

The Moutard transformation provides a Darboux-type transformation of the asymptotic lattices and defines the discrete $\mathrm{W}$-congruences [5].

Definition 4. By a discrete $W$-congruence we mean a two-parameter family of straight lines connecting two asymptotic lattices in such a way that the lines are tangent to the lattices in corresponding points.

It turns out that any discrete $\mathrm{W}$-congruence can be obtained via a Moutard transformation [5].

Proposition 3. Given a discrete $W$-congruence connecting $\boldsymbol{x}$ and $\widehat{\boldsymbol{x}}$, then the normal vectors $\boldsymbol{N}$ and $\widehat{\boldsymbol{N}}$ which define $\boldsymbol{x}$ and $\widehat{\boldsymbol{x}}$ via the Lelieuvre formulas, are related by a Moutard transformation.

In the line-geometric approach the lines of the $\mathrm{W}$-congruence are represented by bivectors

$$
\mathfrak{q}=\left(\begin{array}{c}
\boldsymbol{x} \\
1
\end{array}\right) \wedge\left(\begin{array}{c}
\Theta N \times \boldsymbol{N} \\
0
\end{array}\right)=A \mathfrak{p}_{1}+B \mathfrak{p}_{2} .
$$

On can show that $\mathfrak{q}$ satisfies the Laplace equation [5].

Theorem 4. Discrete $W$-congruences are represented by two dimensional quadrilateral lattices in the Plücker quadric $\mathcal{Q}_{P}$. 


\section{Acknowledgements}

I would like to thank the organizers of the NEEDS'99 Workshop for invitation and support, and the Warsaw Scientific Society for supporting my travel.

\section{References}

[1] Bobenko A and Pinkall U, Discrete Isothermic Surfaces, J. Reine Angew. Math., 1996, V.475, $187-208$.

[2] Bobenko A and Pinkall U, Discrete Surfaces with Constant Negative Gaussian Curvature and the Hirota Equation, J. Diff. Geom., 1996, V.43, 527-611.

[3] Bobenko A and Schief W K, Discrete Indefinite Affine Spheres, in Discrete Integrable Geometry and Physics, Editors A Bobenko and R Seiler, Clarendon Press, Oxford, 1999, 113-138.

[4] Cieśliński J, Doliwa A and Santini P M, The Integrable Discrete Analogues of Orthogonal Coordinate Systems are Multidimensional Circular Lattices, Phys. Lett. A, 1997, V.235, 480488.

[5] Doliwa A, Discrete Assymptotic Nets and W-Congruences within the Plücker Geometry, solv-int/9909015.

[6] Doliwa A, Geometric Discretisation of the Toda System, Phys. Lett. A, 1997, V.234, 187-192.

[7] Doliwa A, Discrete integrable geometry with ruler and compass, in Symmetries and Integrability of Difference Equations, Editors P Clarkson and F Nijhoff, Cambridge University Press, 1999, 122-136.

[8] Doliwa A, Quadratic Reductions of Quadrilateral Lattices, J. Geom. Phys., 1999, V.30, 169186.

[9] Doliwa A, Manakov S V and Santini P M, $\bar{\partial}$-Reductions of the Multidimensional Quadrilateral Lattice: the Multidimensional Circular Lattice, Comm. Math. Phys., 1998, V.196, 1-18.

[10] Doliwa A and Santini P M, The Symmetric and Egorov Reductions of the Quadrilateral Lattice, solv-int/9907012.

[11] Doliwa A and Santini P M, Multidimensional Quadrilateral Lattices are Integrable, Phys. Lett. A, 1997, V.233, 365-372.

[12] Doliwa A, Santini P M and Mañas M, Transformations of Quadrilateral Lattices, solvint/9712017, J. Math. Phys. (to appear).

[13] Eisenhart L P, A Treatise on the Differential Geometry of Curves and Surfaces, Ginn and Company, Boston, 1909.

[14] Klein F, Vorlesungen Über Höhere Geometrie, Springer, Berlin, 1926.

[15] Konopelchenko B G and Pinkall U, Projective Generalizations of Lelieuvre's Formula, Sfb 288 Preprint No. 333, math.DG/9807083, to appear in Geometriae Dedicata.

[16] Konopelchenko B G and Schief W K, Three-Dimensional Integrable Lattices in Euclidean Spaces: Conjugacy and Orthogonality, Proc. Roy. Soc. London A, 1998, V.454, 3075-3104.

[17] Mañas M, Doliwa A and Santini P M, Darboux Transformations for Multidimensional Quadrilateral Lattices. I, Phys. Lett. A, 1997, V.232, 99-105.

[18] Nieszporski M, On Discretization of Asymptotic Nets (to be published).

[19] Nimmo J J C and Schief W K, Superposition Principles Associated with the Moutard Transformation. An Integrable Discretisation of a $(2+1)$-Dimensional Sine-Gordon System, Proc. R. Soc. London A, 1997, V.453, 255-279.

[20] Sauer R, Differenzengeometrie, Springer, Berlin, 1970. 